\title{
SARS-CoV-2 Wastewater Surveillance for Public Health Action: Connecting Perspectives from Wastewater Researchers and Public Health Officials During a Global Pandemic
}

Jill S. McClary-Gutierrez $z^{1} \dagger$, Mia C. Mattioli ${ }^{2}$, Perrine Marcenac ${ }^{2}$, Andrea I. Silverman ${ }^{3}$, Alexandria B. Boehm ${ }^{4}$, Kyle Bibby ${ }^{5}$, Michael Balliet ${ }^{6}$, Francis L. de los Reyes III ${ }^{7}$, Daniel Gerrity ${ }^{8}$, John F. Griffith ${ }^{9}$, Patricia A. Holden ${ }^{10}$, Dimitrios Katehis ${ }^{11}$, Greg Kester ${ }^{12}$, Nathan LaCross ${ }^{13}$, Erin K. Lipp ${ }^{14}$, Jonathan Meiman ${ }^{15}$, Rachel T. Noble ${ }^{16}$, Dominique Brossard ${ }^{17 *}$, Sandra L. McLellan ${ }^{1 *}$

${ }^{1}$ School of Freshwater Sciences, University of Wisconsin-Milwaukee, Milwaukee, WI, USA

${ }^{2}$ Waterborne Disease Prevention Branch, Division of Foodborne, Waterborne, and

Environmental Diseases, National Center for Emerging and Zoonotic Infectious Diseases, Centers for Disease Control and Prevention, USA

${ }^{3}$ Department of Civil and Urban Engineering, New York University Tandon School of Engineering Brooklyn, NY, USA

${ }^{4}$ Department of Civil and Environmental Engineering, Stanford University, Stanford, CA, USA ${ }^{5}$ Department of Civil and Environmental Engineering and Earth Sciences, University of Notre Dame, Notre Dame, IN, USA

${ }^{6}$ County of Santa Clara, Department of Environmental Health, San Jose, CA, USA

${ }^{7}$ Department of Civil, Construction, and Environmental Engineering, North Carolina State University, Raleigh, NC, USA

${ }^{8}$ Applied Research and Development Center, Southern Nevada Water Authority, Las Vegas, NV, USA

${ }^{9}$ Southern California Coastal Water Research Project, Costa Mesa, CA, USA

${ }^{10}$ Bren School of Environmental Science \& Management, University of California, Santa Barbara, CA

${ }^{11}$ New York City Department of Environmental Protection, New York, NY, USA

${ }^{12}$ California Association of Sanitation Agencies, Sacramento, CA, USA

${ }^{13}$ Utah Department of Health, Salt Lake City, UT, USA

${ }^{14}$ Department of Environmental Health Science, University of Georgia, Athens, GA, USA

${ }^{15}$ Wisconsin Department of Health Services, Madison, WI, USA

${ }^{16}$ Institute of Marine Sciences, University of North Carolina at Chapel Hill, Morehead City, NC, USA

${ }^{17}$ Department of Life Sciences Communication, University of Wisconsin-Madison, and Morgridge Institute for Research, Madison, WI, USA

*Correspondance: Dominique Brossard dbrossard@wisc.edu; Sandra L. McLellan mclellan@uwm.edu

†Present Address: Department of Civil and Environmental Engineering and Earth Sciences, University of Notre Dame, Notre Dame, IN, USA 


\begin{abstract}
Wastewater surveillance for SARS-CoV-2 has garnered extensive public attention during the COVID-19 pandemic as a proposed complement to existing disease surveillance systems. Over the past year, environmental microbiology and engineering researchers have advanced methods for detection and quantification of SARS-CoV-2 viral RNA in untreated sewage and demonstrated that the trends in wastewater are correlated with trends in cases reported days to weeks later depending on the location. At the start of the pandemic, the virus was also detected in wastewater in locations prior to known cases. Despite the promise of wastewater surveillance, for these measurements to translate into useful public health tools, it is necessary to bridge the barriers between researchers and the public health responders who will ultimately use the data. Here we describe the key uses, barriers, and applicability of SARS-CoV-2 wastewater surveillance for supporting public health decisions and actions. This perspective was formed from a multidisciplinary group of environmental microbiology, engineering, wastewater, and public health experts, as well as from opinions shared during three focus group discussions with officials from ten state and local public health agencies. The key barriers to use of wastewater surveillance data identified were: (1) As a new data source, most public health agencies are not yet comfortable interpreting wastewater data; (2) Public health agencies want to see SARS-CoV-2 wastewater data in their own communities to gain confidence in its utility; (3) New institutional knowledge and increased capacity is likely needed to sustain wastewater surveillance systems; and (4) The ethics of wastewater surveillance data collection, sharing, and use are not yet established. Overall, while wastewater surveillance to assess community infections is not a new idea, by addressing these barriers, the COVID-19 pandemic may be the initiating event that turns this emerging public health tool into a sustainable nationwide surveillance system.
\end{abstract}

\title{
Keywords
}

SARS-CoV-2, wastewater surveillance

\section{Introduction}

SARS-CoV-2 wastewater surveillance is rapidly evolving as a public health tool that holds both promise and challenges ${ }^{1,2}$. In concept, a sewer system contains biological waste from the human population it serves. Biological constituents, including pathogens, enter the sewer system through feces, urine, saliva, and other excreta, and the pathogen concentrations represent input from the human population served by the sewer network (termed the "sewershed") ${ }^{3}$. Given that SARS-CoV-2 RNA is shed in human feces of asymptomatic and symptomatic infections ${ }^{4,5}$, the potential for COVID-19 community-level surveillance through wastewater has garnered much attention since the first report of detection of the SARS-CoV-2 RNA in wastewater in March $2020^{6}$.

SARS-CoV-2 wastewater surveillance could be an important complement to existing public health surveillance for the COVID-19 response as it has the ability to provide information on the 
infection trends in newly reported cases in a community without being influenced by availability of and access to clinical testing resources or healthcare-seeking behavior ${ }^{7-9}$. However, there are practical and technological challenges to implementing and interpreting this new surveillance tool. Precisely measuring levels of virus in a complicated wastewater matrix requires specialized equipment and expertise, and quality controls and quality assurance procedures distinct from clinical testing are necessary for precise molecular quantification ${ }^{10}$.

During the pandemic, there has been a tremendous effort by the science and engineering research communities and commercial laboratories to develop SARS-CoV-2 RNA detection and quantification methods for wastewater surveillance ${ }^{2,11,12}$. As a result of these concerted efforts, SARS-CoV-2 RNA concentrations are now being measured in many wastewater systems globally, and the data are showing wastewater viral RNA concentration trends are correlated with trends in new cases reported days to weeks later depending on reporting lags s, $^{1,7-15}$. Some public health managers are already integrating these data into their COVID-19 response decision-making processes ${ }^{16}$.

Despite the technological advances, there are barriers to using wastewater surveillance data to inform public health decisions. Notably there is a communication gap about how to utilize wastewater surveillance data between researchers and engineers quantifying SARS-CoV-2 RNA in wastewater and the public health practitioners tasked with incorporating information from wastewater data with data from other surveillance sources, such as reported COVID-19 cases, hospitalizations, and deaths. Bridging the gap between research groups generating wastewater surveillance data and the public health sector may help to harness the long-term potential of SARS-CoV-2 wastewater surveillance as a tool for public health disease surveillance and decision-making.

In an effort to bridge this identified gap, the Sloan Foundation supported a group of academic investigators to convene an interdisciplinary expert group with the objective of facilitating conversations around the current opportunities, limitations, and challenges of using SARS-CoV2 wastewater data in public health action.

Common definitions of wastewater surveillance terminology are given in Box $1^{8}$. 


\section{Box 1: Key Wastewater Surveillance Terms}

Grab samples: Samples collected as a single "grab" volume at a single point in time

Composite samples: Samples collected by pooling multiple grab samples over a set time period. Flow-weighted composite samples are pooled after set flow intervals (e.g., one subsample per 200,000 gallons of flow); composite samplers refer to instruments used to automatically collect and pool sample volumes at specific intervals in order to create a composite sample

Sewer transit time: The average time for sewage to travel from an upstream source (e.g., toilet flush) to a downstream sampling point (e.g., treatment plant)

Solids: The nonaqueous fraction of sewage, which may be in the untreated sewage sample or accumulated during the treatment process

Method controls: A range of additional measurements needed to ensure method integrity and appropriate interpretation of SARS-CoV-2 RNA concentration data, including a matrix recovery control, human fecal normalization, quantitative measurement controls, and controls to assess molecular method inhibition

Replication: The same procedure performed multiple times to assess precision of the measurement

Uncertainty: Uncertainty can refer to unknown relationships between a measurement and another metric, such as diagnosed cases. Uncertainty can also be introduced because of variability in measurements due to representative sampling, technical precision, or instrument error.

\section{Interdisciplinary Focus Group Discussions}

An interdisciplinary group of experts in environmental virology, environmental microbiology, wastewater engineering, and public health was formed. The group consisted of ten academic or research institutions, two wastewater agencies, and one city environmental department, together representing a cross section of U.S. institutions involved in SARS-CoV-2 wastewater research. The public health representatives in the group included practitioners from one county and two states health departments that had already begun to develop or implement wastewater surveillance programs in their jurisdictions as part of their COVID-19 response. 
In November 2020, the expert group convened three focus groups to better understand current perspectives of public health responders on the barriers to using SARS-CoV-2 wastewater surveillance data and how wastewater data could support local public health decisions during the rapidly evolving pandemic. Divided into three separate virtual meetings, the focus groups included expert group members and officials from two additional wastewater utilities and one city, one district, three county, and three state public health departments from urban and rural communities. Moreover, epidemiological and laboratory lead staff from the CDC National Wastewater Surveillance System (NWSS) participated in the focus groups ${ }^{8}$. Considering the sensitivity of response-related data and resulting public health action for COVID-19, focus groups were not recorded to allow for open discussion of data interpretation and challenges. Attendee responses were summarized and reviewed by expert group members without the use of analytical software. The University of Wisconsin-Milwaukee Institutional Review Board review of this project granted it category 2 exempt status (21.132). Informed consent was obtained from all focus group attendees.

Based on the results of the focus groups, we provide the major barriers identified by public health officials for implementing and using data from wastewater-based infectious disease surveillance programs. We also highlight methodological best practices for wastewater researchers and testers to facilitate utilization of wastewater data by public health officials, and finally, we point toward critical actions needed by both wastewater surveillance method developers and surveillance program implementers in order to effectively incorporate wastewater surveillance into the COVID-19 public health response. These findings are summarized in Table 1.

\section{Barrier 1. As a new data source, most public health agencies are not yet comfortable interpreting wastewater data.}

During focus group discussions, 58\% (7/12) of public health representative focus group participants reported that personnel and resources are stretched well past capacity, resulting in a limited ability to incorporate new and unfamiliar metrics into the workload, especially without demonstration of their value in decision making. Unlike case counts or hospitalizations that have a relationship to disease in the community, wastewater surveillance data are presented as concentrations of SARS-CoV-2 gene copies per volume of wastewater (commonly expressed as per liter of sewage or per gram of solids), which may be difficult for people unfamiliar with the measurement to contextualize, leading to challenges in interpreting the data and results. The reporting of wastewater data can be even further complicated because, in order to compare across time and space, the wastewater data are often normalized by total daily wastewater flow (expressed as SARS-CoV-2 gene copies per day) or by the concentration of a human-specific gut microbe ${ }^{17}$. Our focus groups identified several additional reasons for the hesitation in using wastewater data for public health responses, which can be grouped into two main categories:

1. Uncharacterized sources of uncertainty and variability: Many factors can influence SARS-CoV-2 RNA concentrations measured in wastewater, such as sampling location, sampling methods (e.g., grab versus flow-weighted composite samples), sewer transit time, the addition of industrial waste or stormwater to the sewer, wastewater flow rates, and fecal shedding rates. These factors are not currently well-defined, and their effects 
on SARS-CoV-2 RNA concentrations are still being investigated; therefore, the degree of natural variability and acceptable uncertainty in the data-factors that are known and incorporated into other surveillance systems—are not known for wastewater surveillance.

2. Lack of methodological standardization: Public health laboratories are accustomed to testing samples using highly standardized methodology with defined levels of uncertainty. The variability in SARS-CoV-2 RNA wastewater measurements that are introduced when concentrating the virus from a large volume, or during RNA extraction or RNA quantification, is not well-defined at this time. In addition, there is no single standard method for concentrating and measuring SARS-CoV-2 RNA from wastewater; in fact, a single method might not be appropriate for all wastewater sources because wastewater composition varies across locations. Each method may be associated with different levels of uncertainty and variability that must be defined using appropriate preanalytical and analytical method controls and replication.

Delineating sources of data uncertainty, defining variability in measurement, and standardizing methodology represent important avenues of inquiry for the research community. In the meantime, many different entities are generating SARS-CoV-2 wastewater data, and these results, coupled with explanations of data limitations and known sources of variability, may facilitate assimilation as public health agencies become more accustomed to the data. In addition, close collaboration between groups generating wastewater data and public health agencies, wastewater utilities, and experts in communication and data visualization will ensure that findings are appropriately communicated to data end-users to prevent false assumptions and under- or over-interpretation. Environmental health departments may be good liaisons between different wastewater surveillance partners because even though they may not be located within the public health department, they often have both extensive public health and wastewater knowledge.

Barrier 2. Public health agencies want to see SARS-CoV-2 wastewater data in their own communities to gain confidence in its application and utility.

Agencies reported significant benefit from seeing the data in action in their own communities, allowing them to gain a greater understanding of the data and its potential value. This is because every community has unique infrastructure, demographics, and public health capacity and challenges that will inevitably influence how SARS-CoV-2 RNA wastewater measurements can be used. Wastewater data can be collected at three different scales ${ }^{18}$ :

1. Wastewater treatment plant: Wastewater sampling routinely occurs at wastewater treatment plants for permit compliance requirements, so additional sampling at the plant is usually straightforward to implement. Wastewater treatment plants can serve "sewersheds" containing thousands to millions of people, depending on their size, and measurements of SARS-CoV-2 RNA at the plant can provide insight into infection burdens in the sewershed population. Depending on the data needs of a wastewater surveillance effort, sampling a smaller geographic area than the sewershed may be needed. 
2. Sub-sewershed: It is possible to sample wastewater from the pipe network that moves waste from households and businesses to the wastewater plant, thus isolating a "subsewershed" population. Collecting samples from within the pipe network is complicated by various factors including lack of adequate maps and challenging access to manholes. Depending on the wastewater infrastructure design and equipment resources, sampling at the sub-sewershed scale can be resource-intensive, and appropriate sampling schemes for the approach presently lack validation.

3. Facility-Level: Information on COVID-19 infections of individuals working and living in individual facilities can potentially be obtained by testing wastewater from the facilities (for example, hospitals, skilled nursing facilities, schools, or universities). Drawings of facilities' plumbing will be necessary to identify potential sampling locations; and intermittent use of water within the facilities will result in intermittent flow in the plumbing, which can challenge sampling efforts ${ }^{19}$. Wide-scale facility-level wastewater surveillance might not be feasible with hundreds of facilities in large counties. Wastewater testing may give a false negative result when cases are present because of difficulty in obtaining a representative sample, inconsistent (or absent) viral shedding in feces by infected individuals, or low sensitivity in the method. Routine screening of individuals may allow for more immediate isolation of cases and contract tracing.

It is important to note that across the US, $80 \%$ of the population is served by a piped sewage network, while the remaining use cesspools or septic systems ${ }^{20}$. There is little evidence supporting the utility of sewage surveillance in these onsite systems.

While seeing the application of wastewater SARS-CoV-2 data in public health practitioners' own communities cannot be overstated, clear case examples of other uses of wastewater data and resulting public health impacts across different jurisdictions and areas were noted as critical for gaining confidence in these novel surveillance data. Some communities have been generating wastewater SARS-CoV-2 datasets since early in the pandemic, giving them the ability to perform retrospective analysis to demonstrate whether SARS-CoV-2 wastewater data effectively captured reported case trends and/or filled gaps in case trends in areas with more limited clinical testing. Below are specific examples provided by public health implementers from the expert group of how wastewater data were used to support their COVID-19 response (Box 2). 
Box 2. Examples provided by public health implementers of how wastewater data were used to support their COVID-19 response.

Wisconsin - The Wisconsin Department of Health Services (WI DHS) initiated a statewide SARS-CoV-2 wastewater testing program in collaboration with the Wisconsin State Laboratory of Hygiene and the University of Wisconsin-Milwaukee. To date, this program has monitored SARS-CoV-2 RNA concentrations once or twice per week in samples collected from 70 municipal wastewater treatment plants that provide service for approximately $53 \%$ of the state's population. Sample collection for select locations began in August 2020 and captured the pre-Thanksgiving surge in COVID-19 cases in northeastern Wisconsin. By including a large number of wastewater treatment plants of various sizes in the program, WI DHS is able to assess correlations between SARS-CoV-2 concentrations in wastewater and diagnosed COVID-19 cases and hospitalizations and identify factors that influence these relationships. Data are publicly available (https://www.dhs.wisconsin.gov/covid-

19/wastewater.htm). Local health departments have used these data to confirm health trends identified through clinical testing, particularly in rural areas of the state with limited testing access. A short turnaround time for wastewater analysis will allow WI public health officials to identify regions with increasing SARS-CoV-2 transmission and anticipate surges in COVID-19 hospitalizations.

Utah - Utah's SARS-CoV-2 wastewater monitoring program began with a limited pilot project in March 2020 as a collaboration between the Utah Department of Environmental Quality (UDEQ), Utah Department of Health (UDOH), and four academic laboratories. Upon successful completion of the pilot, sampling was extended in July 2020 to wastewater facilities statewide. As of January 2021, the program collects samples twice a week from 33 facilities that serve approximately $87 \%$ of the state's population. Utah developed a public dashboard (wastewatervirus.utah.gov), integrated the data into a restricted access UDOH internal dashboard, and currently disseminates a summary of new data several times a week to local health departments, UDOH leadership, and other pandemic response personnel. To date, wastewater surveillance data have been used to help direct clinical testing resources (particularly mobile testing teams) to areas with low prevalence of clinical testing, determine where to send mask-wearing compliance observers, and assist the interpretation of other surveillance data. As an example, in July 2020, the wastewater surveillance data indicated declining case rates in some regions of the state. However, the number of people being tested was also decreasing in some of these areas, raising the possibility that the declining case rates were artifacts of clinical testing efforts. Consistently decreasing SARSCoV-2 RNA concentrations in wastewater were able to support the conclusion that the observed declining case rates were real. Wastewater data were a leading indicator for reported cases, with sewershed-associated case rates showing trends 4-7 days after wastewater levels. 


\section{Box 2. Examples provided by public health implementers of how wastewater data were used to support their COVID-19 response.}

Santa Clara County, California - The County of Santa Clara Emergency Operations Center and Public Health Department serve a population of almost two million residents and engaged in early evaluation of wastewater surveillance for SARS-CoV-2 in partnership with Stanford University researchers. Using a multidisciplinary team, a monitoring approach was developed to analyze SARS-CoV-2 RNA in settled solids at all four wastewater treatment plants in the County, comprising over $95 \%$ of the County's total population. A pilot project involving four regional wastewater treatment plants provided daily measurements with a 24-hour turnaround time. This fast turnaround allows County officials to see trends in wastewater data before receipt of clinical data because of lags in the reporting of clinical test results (5-14 days in late 2020 through early 2021). The County and their partners continue to evaluate wastewater surveillance data in conjunction with other public health data to better understand the COVID-19 trends as well as limitations in interpretation of wastewater data. As an example, the County has observed trends in measured SARS-CoV-2 RNA from the wastewater surveillance to generally track with positive COVID-19 case data in the four sewersheds being evaluated. The County will continue to evaluate the data over the next several months to determine if additional trends can be identified and to understand what public health actions might be implemented in response.

\section{Barrier 3. New institutional knowledge and investment in resources and personnel are needed to sustain wastewater surveillance systems.}

Current efforts to monitor wastewater for SARS-CoV-2 have developed in an ad hoc manner during an active pandemic. The environmental virology equipment required for sample processing are not typically available in public health or wastewater laboratories. Because of this, many academic research laboratories are conducting the laboratory analysis for current surveillance programs. However, this approach will likely not be sustainable and instead necessitates transfer of these functions to municipal, public health, or commercial laboratories. Wastewater experts can assist in this transition by partnering with local laboratories and promoting data and methods sharing across the academic, wastewater, and public health sectors. Transferring technical knowledge between researchers and laboratories implementing these methods will ideally occur early during program implementation. As an example, in establishing their SARS-CoV-2 wastewater monitoring program, the New York City Department of Environmental Protection (NYC DEP) engaged academic partners at New York University, Queens College, and Queensborough Community College. All methodological development work for the NYC DEP program occurred in the City's own laboratory with academic partners and NYC laboratory analysts working side-by-side in methodological optimization and implementation. This allowed multidirectional and workforce capacity building and exchange of technical information, ultimately resulting in an ongoing and self-sufficient wastewater 
monitoring program in NYC. These types of stakeholder relationships can aid widespread implementation in the county and state.

Central to our discussions with public health and wastewater practitioners was an overwhelming desire for an improved organizational structure between the various stakeholders needed to conduct a wastewater surveillance program. Municipal wastewater agencies are actively engaged in public health disease prevention by treating wastewater. However, they rarely have active working relationships with public health agencies nor are they often engaged in infectious disease response efforts. These challenges can limit the efficiency of implementing a surveillance program.

Academic researchers partnered with wastewater and public health agencies to launch current wastewater surveillance efforts. As methods evolve, communication with these new partners in infectious disease response can identify needs, facilitate knowledge transfer, and build longer term relationships to promote partner-driven research. Investments in physical laboratory capacity, personnel, and interagency collaboration frameworks to build this new institutional knowledge into public health surveillance frameworks for future epidemics can ensure that these partnerships are valuable in the long term. CDC NWSS is advancing these efforts by developing national data reporting standards and analytics systems, as well as supporting state, local, and territorial capacity building necessary to ensure a sustainable and efficient public health surveillance system ${ }^{8}$.

\section{Barrier \#4: The ethics of wastewater surveillance data collection, sharing, and use are not yet established.}

Wastewater SARS-CoV-2 RNA concentration data collected in appropriately large sewersheds are not individually identifiable, but concerns over stigma or privacy may occur if collecting samples from a sufficiently small population or specific community when individuals maybe identified through deductive disclosure ${ }^{1}$. Some public health agencies and wastewater utilities are therefore hesitant to engage with wastewater surveillance data because of a lack of clarity over privacy, confidentiality, regulatory, and ethical issues and concerns. Public health agencies are entrusted to protect the broader public, and therefore must ensure that their efforts are not inadvertently leaving out or inappropriately targeting certain demographic groups because of infrastructure access or design constraints. In contrast to healthcare data, environmental monitoring data are typically not considered a protected data type, and this disconnect represents an additional challenge to integrating wastewater data into public health data streams. As genomic sequencing approaches are applied to wastewater surveillance to evaluate emerging variants ${ }^{21}$, methods that inventory the total genetic signal, such as metagenomics, also have the potential to contain identifiable personal genetic information. Data reporting standards could require exclusion of human genetic information and wastewater sample location information.

Efforts are underway by both the research and governmental communities to evaluate the ethics and privacy limitations for wastewater surveillance data. While ensuring the ethical use of this data is paramount, wastewater surveillance data may be uniquely able to address some of the 
inadvertent biases of other public health surveillance systems that depend on healthcare access and health-seeking behaviors.

\section{Conclusions}

SARS-CoV-2 wastewater data have added value as a biologically independent, passive source of data that public health agencies can take advantage of for the COVID-19 pandemic response. As research on wastewater testing for SARS-CoV-2 continues, the methods used to generate and analyze these data are expected to improve and reduce the uncertainties associated with this new data source. For widespread adoption as a public health tool, two-way communication and knowledge co-development may ensure that wastewater data have clear value in addressing public health needs, are simple to integrate into other surveillance and health systems, and are used for public health decisions and actions. The COVID-19 pandemic may be the motivating event for creating a sustainable structure to support wastewater surveillance as a unique approach for community-level health monitoring purposes. Investments in resources and personnel can create and sustain a robust wastewater surveillance system for current and future public health emergencies and maintain relationships among stakeholders involved in wastewater surveillance programs. Such investments will continue to build institutional knowledge to support the integration of wastewater data into existing surveillance frameworks for public health actions.

\section{Disclaimer}

The contents of this paper are solely the responsibility of the authors and do not necessarily represent the official views of the US Centers for Disease Control and Prevention.

\section{Acknowledgments}

Funding was provided by the Alfred P. Sloan Foundation to authors SLM, AIS, ABB, KB, and DB. We thank Catherine Hoar, Marlene Wolfe, and Aaron Bivins for contributions to this project. We also focus group participants that included individuals from Northeast Georgia Health District, North Carolina Department of Health and Human Services, Jackson County Department of Public Health, California Department of Public Health, Orange County Sanitation District, Miami-Dade Water \& Sewer Department, Cambridge Public Health Department, and Mathematica Policy Research.

\section{Conflict of Interest}

The authors declare no conflict of interest

\section{References}

1. Polo D, Quintela-Baluja M, Corbishley A, et al. Making waves: Wastewater-based epidemiology for COVID-19 - approaches and challenges for surveillance and prediction. Water Research 2020; 186:116404.

2. Bivins A, North D, Ahmad A, et al. Wastewater-Based Epidemiology: Global Collaborative to Maximize Contributions in the Fight Against COVID-19. Environmental Science \& Technology 2020; 54:7754-7. 
3. Tchobanoglus G, Burton F, Stensel HD. Wastewater engineering: Treatment and reuse. American Water Works Association Journal 2003; 95(5): 201.

4. Gao QY, Chen YX, Fang JY. 2019 Novel coronavirus infection and gastrointestinal tract. Journal of Digestive Diseases 2020; 21:125-6.

5. Zhang W, Du R-H, Li B, et al. Molecular and serological investigation of 2019-nCoV infected patients: implication of multiple shedding routes. Emerging Microbes and Infections 2020; 9:386-9.

6. Medema G, Heijnen L, Elsinga G, Italiaander R, Brouwer A. Presence of SARSCoronavirus-2 RNA in sewage and Correlation with Reported COVID-19 Prevalence in the Early Stage of the epidemic in The Netherlands. Environmental Science \& Technology Letters 2020; 7:511-6.

7. Graham KE, Loeb SK, Wolfe MK, et al. SARS-CoV-2 RNA in Wastewater Settled Solids Is Associated with COVID-19 Cases in a Large Urban Sewershed. Environmental Science and Technology 2021; 55:488-98.

8. Centers for Disease Control and Prevention. National Wastewater Surveillance System (NWSS). 2020. https://www.cdc.gov/coronavirus/2019-ncov/cases-updates/wastewatersurveillance.html (accessed 22 March 2021).

9. Weidhaas J, Aanderud ZT, Roper DK, et al. Correlation of SARS-CoV-2 RNA in wastewater with COVID-19 disease burden in sewersheds. Sci Total Environ 2021; 775: 145790.

10. The Water Research Foundation. Wastewater Surveillance of the COVID-19 Genetic Signal in Sewersheds: Recommendations from Global Experts. In: International Water Research Summit on Environmental Surveillance for the Genetic Signal of SARS-CoV-2 in Sewersheds. 2020.

11. Wigginton KR, Boehm AB. Environmental Engineers and Scientists Have Important Roles to Play in Stemming Outbreaks and Pandemics Caused by Enveloped Viruses. Environ Sci Technol 2020; 54: 3736-9.

12. Pecson BM, Darby E, Haas CN, et al. Reproducibility and sensitivity of 36 methods to quantify the SARS-CoV-2 genetic signal in raw wastewater: findings from an interlaboratory methods evaluation in the U.S. Environ Sci Water Res Technol 2021; 7: 504-520.

13. Gonzalez R, Curtis K, Bivins A, et al. COVID-19 surveillance in Southeastern Virginia using wastewater-based epidemiology. Water Res 2020; 186: 116296.

14. Peccia J, Zulli A, Brackney DE, et al. Measurement of SARS-CoV-2 RNA in wastewater tracks community infection dynamics. Nat Biotechnol 2020; 38: 1164-7.

15. Gerrity D, Papp K, Stoker M, Sims A, Frehner W. Early-pandemic wastewater surveillance of SARS-CoV-2 in Southern Nevada: Methodology, occurrence, and incidence/prevalence considerations. Water Res X 2021; 10: 100086.

16. Stadler LB, Ensor KB, Clark JR, et al. Wastewater Analysis of SARS-CoV-2 as a Predictive Metric of Positivity Rate for a Major Metropolis. medRxiv 2020: 1-36.

17. Centers for Disease Control and Prevention. Wastewater Surveillance Data Reporting \& Analytics. 2020. https://www.cdc.gov/coronavirus/2019-ncov/cases-updates/wastewatersurveillance/data-reporting-analytics.html (accessed 22 March 2021).

18. Centers for Disease Control and Prevention. Developing a Wastewater Surveillance Sampling Strategy. 2020. https://www.cdc.gov/coronavirus/2019-ncov/casesupdates/wastewater-surveillance/developing-a-wastewater-surveillance-samplingstrategy.htm|\#anchor 1602855341209 (accessed 22 March 20210).

19. Gibas C, Lambirth K, Mittal N, et al. Implementing Building Level SARS-CoV-2 Wastewater Surveillance on a University Campus. medRxiv 2021: 1-21.

20. Centers for Disease Control and Prevention and U.S. Department of Housing and Urban Development. Chapter Ten: On-site Wastewater Treatment. In: Healthy housing reference manual. Atlanta: US Department of Health and Human Services, 2006: 10-1-10-2. 
21. Crits-Christoph A, Kantor RS, Olm MR, et al. Genome sequencing of sewage detects regionally prevalent SARS-CoV-2 variants. MBio 2020; 12: e02703-20.

Table 1. Summary of barriers, best practices, and future needs for public health agencies (PHA) using wastewater surveillance data for public health action.

\begin{tabular}{lll}
\hline \multicolumn{1}{c}{ Barriers } & \multicolumn{1}{c}{ Recommended Best Practices } & Future Needs \\
\hline $\begin{array}{l}\text { 1. Many PHA are not yet } \\
\text { comfortable interpreting } \\
\text { wastewater data }\end{array}$ & $\begin{array}{l}\text { alongside data limitations and known } \\
\text { variability sources }\end{array}$ & $\begin{array}{l}\text { Evaluation of wastewater } \\
\text { data variability and } \\
\text { uncertainty sources in a } \\
\text { variety of systems }\end{array}$ \\
& $\begin{array}{l}\text { - Collaborate with laboratories, } \\
\text { wastewater utilities, environmental health } \\
\text { departments, and communications experts }\end{array}$ & \\
\hline $\begin{array}{l}\text { 2. PHA want to see wastewater } \\
\text { data in their own communities } \\
\text { to gain confidence in utility }\end{array}$ & $\begin{array}{l}\text { applications and perspectives } \\
\text { Provide case studies from community }\end{array}$ & $\begin{array}{l}\text { Documentation of } \\
\text { wastewater surveillance } \\
\text { use cases for adoption in } \\
\text { different communities and } \\
\text { infrastructure systems }\end{array}$ \\
\hline
\end{tabular}

3. New knowledge and investment needed to sustain wastewater surveillance systems
- Co-develop programs and methods with scientific experts and government agencies

- Share methods and experiences across academic, wastewater, and public health
Investment in physical lab capacity, personnel, and interagency collaboration frameworks
4. Ethics of wastewater surveillance data sharing and use not yet established
- Evaluate sample anonymity

- Engage the public in collection and data use
Development of ethical wastewater data use standards for surveillance and research 